\title{
Relationship of Proximal Renal Tubular Dysgenesis and Fetal Liver Injury in Neonatal Hemochromatosis
}

\author{
SILVANA F. BONILLA, HECTOR MELIN-ALDANA, AND PETER F. WHITINGTON \\ Departments of Pediatrics [S.F.B., P.F.W.] and Pathology [H.M.-A.], Northwestern University Feinberg School of Medicine, \\ Chicago, Illinois 60614
}

\begin{abstract}
Renal tubular dysgenesis has been reported in isolated cases of neonatal hemochromatosis $(\mathrm{NH})$. We hypothesized that fetal liver injury in $\mathrm{NH}$ impairs proximal renal tubular development via impaired hepatic angiotensinogen (AGT) elaboration. Morphometric analyses were performed of postmortem liver and kidney sections of cases of proven $\mathrm{NH}$ and postconception age-matched controls for renal proximal tubule density, hepatocyte mass, and hepatic AGT expression. Proximal tubule density was markedly reduced in $\mathrm{NH}$ cases, although they showed a spectrum from mild to severe paucity. Hepatic AGT expression was markedly reduced in $\mathrm{NH}$ cases and correlated closely with reduced hepatocyte mass. A linear relationship was established between hepatic AGT expression and the degree of renal tubular dysgenesis suggesting that there is a relationship between them. Our results demonstrate that there is a spectrum of kidney pathology in patients with $\mathrm{NH}$ including a large proportion of cases with severe proximal tubular dysgenesis. Hepatic synthetic failure resulting in insufficient production of AGT to support renal tubular development is the likely mechanism of kidney disease in NH. (Pediatr Res 67: 188-193, 2010)
\end{abstract}

$\mathrm{N}$ eonatal hemochromatosis $(\mathrm{NH})$ is defined by the presence in newborns of liver disease in association with pathologic siderosis of various organs and tissues in a pattern similar to hereditary hemochromatosis (1). NH has also been called "neonatal iron storage disease" and "perinatal hemochromatosis" on the basis of these unique pathologic findings. $\mathrm{NH}$ is invariably associated with severe liver injury, which evidently is initiated during fetal life (2). Current thinking is that gestational alloimmune hepatocyte injury is involved in the pathogenesis of most or all cases of $\mathrm{NH}(3,4)$.

Several clinical observations date the onset of liver injury in $\mathrm{NH}$ to midgestation. One such observation is the postmortem finding of renal tubular dysgenesis reported in five neonates with $\mathrm{NH}$ (5-7). The hallmark histopathologic findings of renal tubular dysgenesis are paucity or absence of proximal tubules and the absence of necroinflammatory disease that could otherwise account for tubular loss. The proximal tubules in this condition appear short and poorly developed, with reduced convolution leading to reduced number of tubular profiles in histologic sections (8). These findings suggest that

Received June 17, 2009; accepted September 18, 2009.

Correspondence: Peter F. Whitington, M.D., Department of Pediatrics, Children's Memorial Hospital, 2300 Children's Plaza, Box 57, Chicago, IL 60614; e-mail: p-whitington@northwestern.edu

Supported by The Children's Memorial Research Center, Chicago, IL; The Children's Liver Research Fund of Children's Memorial Hospital, Chicago, IL; and The Liver Foundation for Kids, Lemont, IL. renal development has been arrested at 20- to 25-wk gestation. Assuming that failed renal development in renal tubular dysgenesis associated with $\mathrm{NH}$ is the result of or a complication of fetal liver injury, the finding dates the liver injury in these cases to around 20-wk gestation (9). That raises the question of whether renal development is affected in more cases of $\mathrm{NH}$ than is reflected in the meager case reports. In addition, the mechanism by which fetal liver injury leads to a rather specific pattern of failed development in the kidney deserves to be addressed. Renal tubular development is dependent on an intact renin-angiotensin system $(10,11)$. Infants born to women receiving angiotensin converting enzyme (ACE) inhibitors demonstrated renal tubular dysgenesis very similar to that seen in the reported cases of $\mathrm{NH}$ (12). The liver is an integral part of the renin-angiotensin system in that it is the site of synthesis of most of all circulating angiotensinogen (AGT) (13) and thus is potentially pivotal in renal development. This study examined the hypothesis that fetal liver injury in NH impairs proximal renal tubular development via impaired hepatic AGT elaboration.

\section{MATERIALS AND METHODS}

Materials were obtained from postmortem examinations of stillbirths and newborns. Cases of $\mathrm{NH}$ were proven by the standard criteria of demonstrated liver disease and extrahepatic siderosis $(1,9)$. $\mathrm{NH}$ cases available to us were examined to select those in which both liver and kidney were available and adequately preserved (i.e. without autolysis) to permit study. Autopsy records of Children's Memorial Hospital and Prentice Women's Hospital were searched to identify possible postconception age-matched controls. These consisted of newborns undergoing postmortem examination for which the autopsy report showed no evidence of liver or kidney disease/injury, either primary or secondary. A case control was selected for each $\mathrm{NH}$ case on the basis of postconception age within $1 \mathrm{wk}$ and samples of liver and kidney that showed adequate preservation. Race and gender were not considered in selecting case controls. This study was approved by the Institutional Review Boards of Children's Memorial Hospital and Northwestern University Feinberg School of Medicine, both by written exemption as it used pre-existing materials from deceased individuals only.

Histology and immunohistochemistry. Paraffin-embedded liver and kidney tissues were sectioned to 5- $\mu \mathrm{m}$ thickness. One section of each was stained with hematoxylin and eosin (H\&E) and Perl's Prussian blue for detection of ferric iron. Using previously reported immunohistochemistry techniques (14), kidney sections were stained for epithelial membrane antigen (EMA; antihuman monoclonal mouse epithelial membrane antigen, Dako Corporation, Carpinteria, CA), which is an antigen known to be confined to the distal tubular epithelium (15), and fumarylacetoacetate hydrolase (FAH; monoclonal antibody kindly provided by RM Tanguay, Quebec Canada), which is confined to the proximal tubular epithelium $(16,17)$, and liver sections were

Abbreviations: EMA, epithelial membrane antigen; FAH, fumarylacetoacetate hydrolase; $\mathbf{N H}$, neonatal hemochromatosis 
stained for AGT (anti-human angiotensinogen polyclonal rabbit serum, Sigma Chemical Co.-Aldrich, St. Louis, MO) and for human IgG (VECTASTAIN ABC kit for Human IgG, Vector Laboratories, Burlingame, CA). Sections were counterstained with hematoxylin. To quantitate apoptosis, TUNEL assay (In Situ Cell Death Detection Kit, Fluorescein; Roche Applied Science, Mannheim, Germany) was performed on kidney specimens from five $\mathrm{NH}$ cases and their case controls. A positive control was included in each group of assays performed for quality control. Digital images of 20 random nonoverlapping fields were obtained for each specimen using a fluorescence microscope and a $20 \times$ objective. Phase contrast images were made to match each fluorescence image to determine the location of TUNEL-positive cells. The number of TUNEL-positive nuclei in epithelial cells in each photograph was counted and averaged to give a TUNEL score for each sample, expressed as number per $\mathrm{mm}^{2}$ of renal cortex.

Density of glomeruli and proximal and distal tubules in renal cortex. Ten nonoverlapping images of the outermost renal cortex (extending from the capsule inward for $1-1.5 \mathrm{~mm}$ ) photographed with a $10 \times$ objective were obtained from each kidney section stained for FAH or EMA. To determine the area density of proximal tubules, FAH-stained tubule profiles were counted, and to determine the area density of distal tubules, EMA-stained (distal) tubules were counted in each photomicrograph. Glomeruli were counted on EMA-stained photomicrographs, where they were clearly visible with the hematoxylin counterstain. The results of 10 photomicrographs from each section were averaged, and the area density of the various elements was expressed as number per $\mathrm{mm}^{2}$ of renal cortex

AGT expression in liver. Liver sections stained for AGT were examined microscopically for distribution of staining. AGT localizes in the cytoplasm of hepatocytes and Kupffer cells (18). Therefore, the photographs of sections used for this analysis included parenchyma only. Five nonoverlapping images were obtained with a $20 \times$ objective of hepatic parenchyma from each liver section stained for AGT. Digital image analysis was used to determine the expression of AGT, as described previously (19). The number of pixels corresponding to AGT were measured based on a narrow band of the brown spectrum corresponding to the stain of clear-cut AGT and computed as a percentage of the total pixels of each image using the Image Processing Tool Kit, version 5.0 (Reindeer Graphics, Asheville, NC). The results of the five fields from each specimen were averaged, and AGT was expressed as area\% for each specimen.

Determination of hepatocyte volume density. Liver sections stained with $\mathrm{H} \& \mathrm{E}$ were analyzed to determine hepatocyte volume density using a point counting method $(20,21)$. Three random nonoverlapping images of hepatic parenchyma from each H\&E-stained sample were obtained with a $40 \times$ objective. Digital images were overlaid with a grid of $13 \times 17$ lines (providing 221 intersections per image), and the position of each intersection was visually determined, whether within the confines of a hepatocyte or not. The tally of points in three images landing within a hepatocyte was divided by the total points (663) to provide a close estimate (error of estimate $<5 \%$ ) of hepatocyte volume density, expressed as volume \%.

Statistical analysis. The findings in NH cases were compared with those in the matched case controls using a paired analysis. A normal distribution of findings was not assumed, and because the hypothesis to be tested was a reduction in proximal tubules in the $\mathrm{NH}$ cases, a one-way, paired Wilcoxon test was used. Relationships between continuous variables within groups were examined using regression analysis. All statistical analysis was performed using the GraphPad Prism 4 statistical package. A $p$ value of 0.05 was considered statistically significant. Group data are presented as mean \pm SD.

\section{RESULTS}

Eleven NH cases were included in the study, each with a postconception age-matched control (Table 1). The postconception ages ranged from 30 to $41 \mathrm{wk}$. Two of the $\mathrm{NH}$ cases were stillborn, whereas none of the case controls were. The causes of death in the $\mathrm{NH}$ cases were liver or multiorgan failure in all. The cause of death in all case controls was perinatal asphyxia.

Selective reduction in proximal tubules in $\mathrm{NH}$. To determine whether the number of proximal tubules counted needed to be adjusted for differences related to the 11-wk span of postconception ages in the subject population (during which total nephrons are known to increase), the glomerular density in the case control samples was plotted against postconception age (Table 1). Linear regression analysis showed no significant relationship between nephron number as represented by glomerular density and postconception age from 30 to $41 \mathrm{wk}$ in the case control samples $\left(\mathrm{r}^{2}=0.112, p=0.314\right)$. Therefore, in subsequent analyses, no adjustment was needed or used. Moreover, the close match of NH cases and case controls by postconception age should compensate in a paired analysis for any possible minor changes in nephron density encountered.

Sections of kidney stained for FAH were analyzed for proximal tubule density, and those stained for EMA were analyzed for distal tubule density and total tubule density. Case controls showed a much greater density of proximal tubules than did $\mathrm{NH}$ cases, whereas the number of distal tubules was similar (Fig. 1). When the area density of proximal tubules was computed, there was clear reduction in the $\mathrm{NH}$ cases relative to their case controls, $18.4 \pm 16.3$ per $\mathrm{mm}^{2}$ versus $76.0 \pm 13.4$ per $\mathrm{mm}^{2}, p=0.0005$ (Fig. 2). Six NH cases showed $<15$ proximal tubules per $\mathrm{mm}^{2}$ of renal cortex, and there was no overlap of the $\mathrm{NH}$ cases with the case controls. The number of distal tubules in the $\mathrm{NH}$ cases $(92.7 \pm$ 26.0 per $\left.\mathrm{mm}^{2}\right)$ and case controls $\left(93.9 \pm 21.0\right.$ per $\left.\mathrm{mm}^{2}\right)$ were not different ( $p=1.034)$ (Fig. 2). In addition, the density of glomeruli was not reduced in $\mathrm{NH}$ relative to the case controls $(p=0.233)$ (Fig. 2). Furthermore, there was no correlation between the density of glomeruli and proximal tubule density in the NH cases $\left(\mathrm{r}^{2}=0.020, p=0.679\right)$ or in the combined

Table 1. The NH cases and their specific case controls

\begin{tabular}{|c|c|c|c|c|c|c|c|c|c|}
\hline $\mathrm{NH}$ case & $\begin{array}{l}\text { PC age } \\
\text { (wk) }\end{array}$ & $\begin{array}{l}\text { Glomeruli number } \\
\text { per } \mathrm{mm}^{2}\end{array}$ & $\begin{array}{l}\text { Prox tub number } \\
\text { per } \mathrm{mm}^{2}\end{array}$ & $\begin{array}{c}\text { AGT } \\
\operatorname{vol}(\%)\end{array}$ & $\begin{array}{c}\mathrm{NH} \\
\text { control }\end{array}$ & $\begin{array}{l}\text { PC age } \\
\text { (wk) }\end{array}$ & $\begin{array}{l}\text { Glomeruli number } \\
\text { per } \mathrm{mm}^{2}\end{array}$ & $\begin{array}{l}\text { Prox tub \# } \\
\text { per } \mathrm{mm}^{2}\end{array}$ & $\begin{array}{c}\text { AGT } \\
\operatorname{vol}(\%)\end{array}$ \\
\hline NH1 & 30 & 11.2 & 13.7 & 9.7 & $\mathrm{CC} 1$ & 30 & 15 & 89.3 & 55.2 \\
\hline $\mathrm{NH} 2$ & 36 & 4.2 & 32 & 1.6 & $\mathrm{CC} 2$ & 36 & 10 & 53.3 & 17.1 \\
\hline NH3 & 32 & 19.6 & 29.6 & 1.4 & $\mathrm{CC} 3$ & 32 & 12 & 60 & 32.9 \\
\hline $\mathrm{NH} 4$ & 35 & 4.1 & 0.8 & 1.4 & $\mathrm{CC} 4$ & 35 & 13.1 & 75.1 & 17.2 \\
\hline NH5 & 38 & 4.5 & 25.7 & 2.0 & CC5 & 37 & 6.5 & 79.6 & 12.6 \\
\hline NH6 & 36 & 5.3 & 8.6 & 4.6 & CC6 & 36 & 15.4 & 73.9 & 64.7 \\
\hline NH7 & 39 & 6.6 & 30.3 & 2.7 & $\mathrm{CC} 7$ & 39 & 17 & 76.6 & 10.7 \\
\hline NH8 & 39 & 9.4 & 4.4 & 3.3 & $\mathrm{CC} 8$ & 39 & 8.9 & 83.5 & 20.1 \\
\hline NH9 & 41 & 10.9 & 50.2 & 2.9 & CC9 & 41 & 23 & 62.9 & 34.9 \\
\hline NH10 & 40 & 11.4 & 2.1 & 2.3 & $\mathrm{CC} 10$ & 40 & 15.2 & 100.1 & 11.8 \\
\hline NH11 & 40 & 28 & 2.5 & 6.9 & $\mathrm{CC} 11$ & 40 & 14.5 & 81.9 & 19.5 \\
\hline
\end{tabular}

PC, postconception age; Prox tub, proximal tubule; AGT, angiotensinogen. 


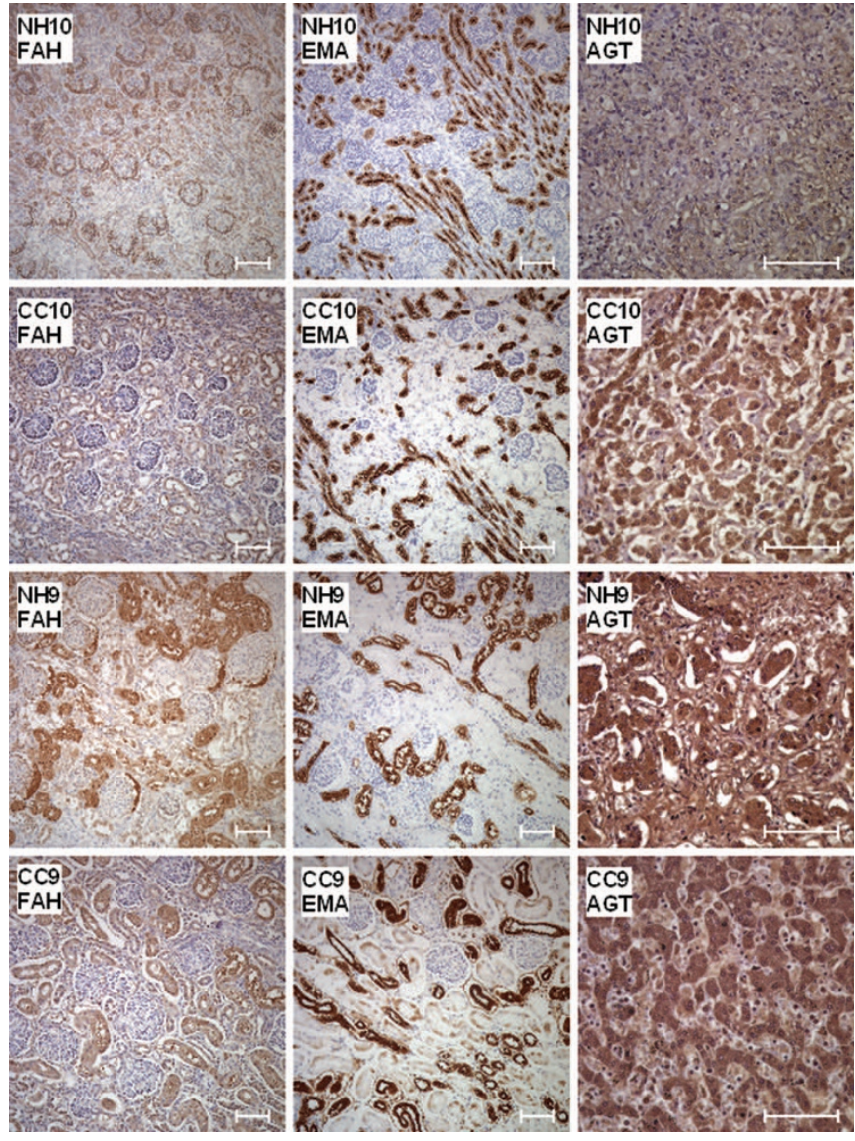

Figure 1. Representative photomicrographs of renal cortex and liver in two cases of $\mathrm{NH}$ and their case controls. The labels refer to specific cases and case controls (Table 1) and staining of kidney for FAH and EMA and liver for AGT. The method produces an intense warm brown stain of cells where the antigen is located. In the NH10 case, almost no tubules stain with FAH, a marker of proximal tubules, whereas in the NH9 case, many more do. This relates to the spectrum of paucity of proximal tubules seen in $\mathrm{NH}$ cases. Case controls all showed FAH positive tubules. Both NH cases showed prominent FAH staining of Bowman's capsule not seen in the controls. NH cases and case controls showed similar numbers of tubules positive for EMA, a marker of distal tubules. The liver of the NH10 case showed few viable hepatocytes and virtually no staining for AGT, whereas the NH9 case showed more recognizable hepatocyte forms (giant cells and pseudorosettes) that stained positively for AGT. The case controls all showed cord patterns of hepatocytes staining positively for AGT. The scale bar in each image is $100 \mu \mathrm{M}$ in length.

$\mathrm{NH}$ and case controls $\left(\mathrm{r}^{2}=0.038, p=0.386\right)$. Thus, the reduction in proximal tubular density seems to be a selective effect of $\mathrm{NH}$ on the kidney. An interesting finding in the $\mathrm{NH}$ cases was FAH staining of Bowman's capsule, which has also been observed in proximal tubular dysgenesis in the twin-twin transfusion syndrome (16). When the proximal tubule fails to develop, the embryologically related parietal epithelium of Bowman's capsule seems to express FAH ectopically.

Renal histopathology was not otherwise different between $\mathrm{NH}$ cases and controls. No subject's kidney showed any degree of inflammation or fibrosis. Examination of Perl's Prussian blue stained sections showed trace amounts of siderosis in tubular epithelium in one $\mathrm{NH}$ case only (data not shown), whereas the remaining 21 specimens showed none. No necrotic tubular epithelial cells could be identified in any case. No apoptotic cells could be identified by standard
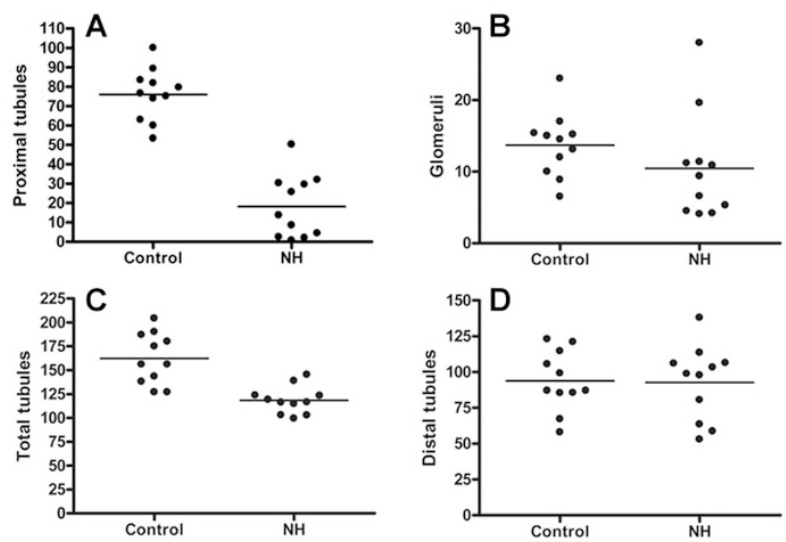

Figure 2. Selective reduction in proximal tubules in $\mathrm{NH}$. The $y$ axis in each panel represents the density of the element expressed as "number per $\mathrm{mm}^{2}$ of renal cortex." Proximal tubule density (Panel $A$ ) is markedly reduced in $\mathrm{NH}$ cases and versus case controls $(p=0.0005)$, whereas glomerular density (Panel B) did not differ in $\mathrm{NH}$ versus case controls. Total tubule density (Panel C) was significantly reduced in NH versus control cases $(p=0.001)$, a difference that was accounted for by the reduced number of proximal tubules in the NH cases because the density of distal tubules (Panel D) did not differ between $\mathrm{NH}$ cases and case controls.

criteria of nuclear condensation and/or fragmentation. We performed TUNEL staining in a subset of five NH cases and their case controls. Small numbers of apoptotic nuclei were identified in tubular epithelium with no difference between $\mathrm{NH}\left(0.74 \pm 0.42\right.$ per $\left.\mathrm{mm}^{2}\right)$ and control cases $(1.02 \pm 0.76$ per $\left.\mathrm{mm}^{2}\right)$.

Relationship of proximal tubular dysgenesis to hepatic AGT. The expression of AGT in the liver was estimated by immunohistochemistry using computerized morphometry. Figure 1 shows representative images from two NH cases and their case controls. AGT expression was primarily localized to hepatocytes in both $\mathrm{NH}$ cases and case controls. In the $\mathrm{NH}$ cases, hepatocytes took many different forms including giant cells and pseudorosettes (Fig. 1), whereas in case controls they appeared in regular cord pattern. The computer imaging analysis system cannot discriminate by location, and thus, the pixels occupied by AGT stain are included, no matter what cell or cell type they occur in. $\mathrm{NH}$ cases had significantly reduced AGT relative to case controls: $3.5 \pm 2.6$ area\% versus $23.2 \pm 17.2$ area\%, $p=0.004$ (Fig. 3). Morphometric analysis showed that $\mathrm{NH}$ cases had marked reduction in hepatocyte volume density versus case controls: $17.5 \pm 5.6$ volume $\%$ versus $66.9 \pm 10.4$ volume $\%, p=0.001$ (Fig. 3). Hepatocyte volume density correlated closely with AGT expression in the combined group of $\mathrm{NH}$ cases and case controls, $\mathrm{r}^{2}=0.527$, $p=0.0003$ (Fig. 3). These data suggest that loss of hepatocyte mass is directly related to reduced AGT expression in NH. Regression analysis demonstrated that hepatic AGT expression correlated directly with proximal tubule density, $\mathrm{r}^{2}=$ $0.344, p=0.004$ (Fig. 3), which provides evidence that there is a relationship between less hepatic AGT expression and low proximal tubule density in $\mathrm{NH}$. 

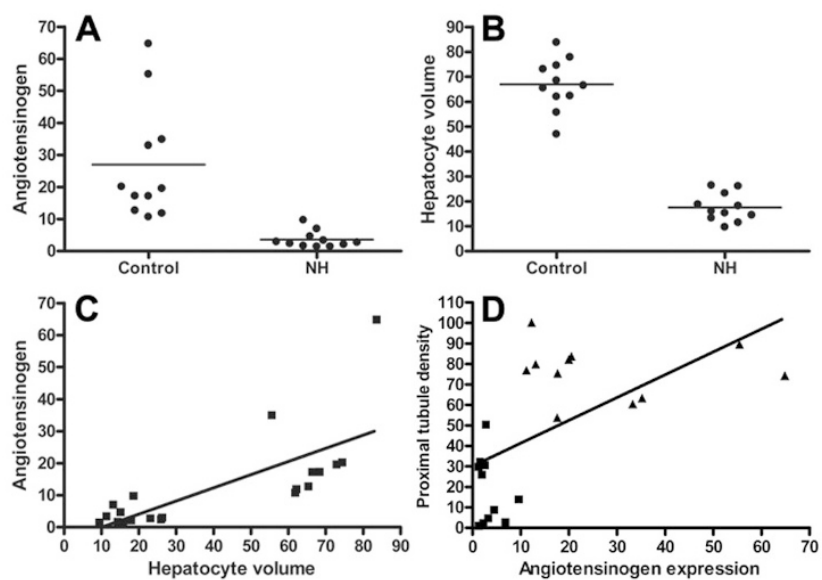

Figure 3. Hepatic AGT expression and its relationship to hepatocyte volume and proximal renal tubule density. AGT is expressed as the percent of hepatic parenchymal volume occupied. Hepatocyte volume is expressed as percent of parenchyma occupied. Proximal tubule density is expressed as number per $\mathrm{mm}^{2}$ of renal cortex. Panel $A$ demonstrates markedly reduced hepatic AGT expression in NH cases versus case controls $(p=0.004)$. Panel $B$ shows that hepatocyte volume density is also reduced in $\mathrm{NH}$ cases relative to case controls $(p=0.001)$, and Panel $C$ demonstrates a close linear correlation between AGT expression and hepatocyte volume density ( $p=0.0003)$. In Panel $D$, proximal tubule density is plotted against hepatic AGT expression. A linear relationship between the two ( $p=0.004)$ suggests a dependence of proximal tubule development on hepatic angiotensinogen ( $\boldsymbol{\square}=\mathrm{NH}$ case; $\boldsymbol{\Delta}=$ case control).

\section{DISCUSSION}

Renal tubular dysgenesis has been reported in only five isolated cases of NH (5-7). Despite the low number of reported cases, finding two such rare conditions in the same patients suggests an association that is not the result of simple coincidence. $\mathrm{NH}$ is a rare disorder that presents sporadically, and thus, experience and expertise in examining affected infants is quite limited. Furthermore, the diagnosis of proximal tubular dysgenesis is not easy, because it requires focused examination and special stains to accomplish. It stands to reason that the association of $\mathrm{NH}$ with renal tubular dysgenesis has been under detected and under reported. Therefore, the first aim of this study was to determine whether $\mathrm{NH}$ related fetal liver disease routinely affects proximal tubule development. Our data suggest that it does and that there is a spectrum of kidney involvement spanning from severe proximal tubule dysgenesis to kidney anatomy that is indistinguishable from normal. The effect of $\mathrm{NH}$ on the kidney seems to be very selective to the proximal tubule. Because of evidence that an intact renin-angiotensin signaling system is required for proximal tubule development, the second aim of this study was to determine whether the link between $\mathrm{NH}$ and proximal renal tubular dysgenesis was failure of hepatic AGT expression. Our data show marked reduction in hepatic AGT expression related to reduced hepatocyte volume in $\mathrm{NH}$ and suggest that this phenomenon is the cause of proximal renal tubular dysgenesis.

Proximal renal tubular dysgenesis is seen primarily out of context with NH (22). Some cases are caused by autosomal recessive inherited gene defects in the renin-angiotensin signaling system, which is critical in renal development (23).
Further evidence of the importance of the renin-angiotensin system in the pathogenesis of proximal tubular dysgenesis comes from the fact that the fetuses of women receiving ACE inhibitors during pregnancy have specific renal abnormalities resembling the genetic disease $(12,24)$. Similar findings have been shown in animal models wherein administration of ACE inhibitors during gestation produces specific renal abnormalities in the fetus (25). The liver is a necessary and integral part of the renin-angiotensin system in that it is the major source of the precursor to angiotensin, AGT. Liver-specific AGT knockouts have insufficient contribution from other sources to support a functioning renin-angiotensin system (13). Hepatic AGT gene expression is responsive to the needs of the developing kidney, being up-regulated during key stages of renal development and in the perinatal period (26). We hypothesized that reduced hepatic synthesis of AGT is the link between $\mathrm{NH}$ and associated proximal renal tubular dysgenesis.

Our results show a markedly reduced hepatic expression of AGT in the NH cases, supporting this hypothesis. Furthermore, our results show a significant relationship between proximal renal tubular density and the expression of AGT in the liver. The NH cases showed a spectrum of tubular dysgenesis, and one case was minimally affected. This may be the result of a time-phase difference between when the effect would take place and when it was being measured. Reduced AGT expression would exert an effect on proximal tubule development throughout the later half of gestation (27), whereas we measured it only at birth. This could lead to some cases in which there is markedly reduced expression at birth, but the hepatic insult may have occurred somewhat late in gestation, and thus, tubule development was affected to a lesser degree. Another possible explanation for the lesser effect seen in some cases is the possibility that different fetal kidneys have differing sensitivity to AGT/angiotensin. For example, in the human case studies where women received ACE inhibitors during gestation, it was difficult to predict from the drug exposure, which fetus would be affected, and only $10 \%$ or so were affected to the degree that renal function was seriously impaired (24). Despite showing severely reduced hepatic AGT expression, no case showed no expression, which would be analogous to the knockout mouse (13) and humans with severe AGT gene mutations (23) where tubule development is uniformly curtailed. The continued expression of some AGT might be expected to support some proximal renal tubule development and lead to near normal tubule numbers in some individuals with greater sensitivity to the stimulus. The specificity of the renal cortical lesion only involving the proximal tubules and the evidence that the renin-angiotensin signaling pathway supports proximal tubule development together with our findings strongly suggests that indeed fetal liver injury in NH leads to impaired development of proximal renal tubules via failure of AGT synthesis.

Alternate explanations for a relationship between fetal liver injury and renal tubular dysgenesis have been offered. Bale $e t$ al.(5) suggested the possibility of renal hypoperfusion secondary to fetal hypotension as a consequence of hepatocellular necrosis. Bernstein and Barajas (28) suggested ischemic loss in cases of proximal tubular dysgenesis not associated with 
NH. They found large amounts of renin in preglomerular arterioles and glomerular mesangial areas, suggesting that renin may have exerted local vasoconstriction with resultant reduced perfusion leading to tubule loss, oligohydramnios, and postnatal oliguria (28). We found no evidence of tubular necrosis or apoptosis in our samples, which suggests failure to develop rather than loss by such a mechanism. Impaired synthesis of growth factors such as transferrin, necessary for renal growth and differentiation (29), or the production of toxic metabolites unable to be cleared by the injured liver are plausible etiologies. Fetal growth in general is affected by $\mathrm{NH}$ (9). This is thought to be a general effect of severe liver disease on somatic growth. However, the very specific renal lesion seen in $\mathrm{NH}$ is more likely to be due to a specific deficiency of a necessary factor such as AGT than to a general effect of liver insufficiency, although it remains to be seen whether one or more cofactors might be related to the severity of expression. Yet, another possibility is that tubule development is impaired via iron toxicity. However, the kidney is not the site of much siderosis in NH (9), and we found trace iron staining in only one of the 11 specimens examined. Furthermore, tissues outside the liver that are affected with iron overload, such as the pancreas and thyroid do not show any anatomic injury or dysgenesis. Therefore, it is unlikely that iron toxicity has anything to do with the proximal renal tubule dysgenesis seen in NH. Finally, because NH is thought to be the manifestation of gestational alloimmune injury to the fetal liver, it is reasonable to consider whether there is some cross-reaction with the developing kidney. We were unable to find any IgG deposits or IgG binding to tubules in the specimens from the NH cases. Additionally, in contrast to the liver, the kidney in these cases shows no injury-no inflammation, no fibrosis, etc - and thus, the paucity of tubules does not seem to result from antibody-mediated destruction of existing tubules (as is evident in hepatocyte loss in the liver). Furthermore, to actually perform a blocking function in the development of fetal disease (as in blocking angiotensin receptors to hypothetically cause failed tubule development), the antibody would need to be in the IgG4 subclass, as only IgG4 engages the $\mathrm{Fc}$ receptor well enough to be escorted across the placenta by FcRn and does not activate complement (30). Thus, a cross-reacting antibody could not do both things: injure the liver and block development of the renal tubule.

The clinical ramifications of our findings are important. More than $50 \%$ of this unselected series of $\mathrm{NH}$ cases had proximal renal tubular dysgenesis to the degree that it would be expected to impair renal function. A near universal finding in $\mathrm{NH}$ is oligohydramnios $(1,9)$, which implies that most cases are affected by some degree of fetal renal insufficiency. All of the live born $\mathrm{NH}$ cases included in this study had a clinical history of oligohydramnios, but it is hard to say that renal insufficiency affected the outcome in this cases, because the materials were all obtained at postmortem examination. However, under certain circumstances, the kidney disease could play an important role in determining outcome. Liver transplantation is a mainstay of treatment of NH. In addition to the stress imposed by this procedure, many of the drugs used afterward are nephrotoxic. It is certainly possible that under- lying proximal tubular dysgenesis could become a dominant problem for the post liver transplant patient with $\mathrm{NH}$ and avoidance of nephrotoxic drugs where possible is recommended. Furthermore, recent data suggest that survival in cases of severe $\mathrm{NH}$ may be significantly improved by treating the immune-mediated liver injury (31). Follow-up studies will be needed to determine whether renal dysfunction persists in cases surviving with or without liver transplantation.

In summary, there is a spectrum of kidney pathology in patients with $\mathrm{NH}$ from mildly affected to severe proximal tubular dysgenesis. Hepatic synthetic failure resulting in insufficient production of AGT to support renal tubular development is the likely mechanism of kidney disease in patients with $\mathrm{NH}$.

Acknowledgments. We thank Dr. William Schnaper for his critical review of this article, Dr. Linda Ernst at Prentice Hospital for her support in getting tissue samples for our control cases, and Sue Kelly R.N. for her help in organizing our materials.

\section{REFERENCES}

1. Knisely AS, Mieli-Vergani G, Whitington PF 2003 Neonatal hemochromatosis Gastroenterol Clin North Am 32:877-889

2. Hoogstraten J, de Sa DJ, Knisely AS 1990 Fetal liver disease may precede extrahepatic siderosis in neonatal hemochromatosis. Gastroenterology 98:16991701

3. Whitington PF 2007 Neonatal hemochromatosis: a congenital alloimmune hepatitis Semin Liver Dis 27:243-250

4. Whitington PF, Kelly S 2008 Outcome of pregnancies at risk for neonatal hemochromatosis is improved by treatment with high-dose intravenous immunoglobulin. Pediatrics 121:e1615-e1621

5. Bale PM, Kan AE, Dorney SF 1994 Renal proximal tubular dysgenesis associated with severe neonatal hemosiderotic liver disease. Pediatr Pathol 14:479-489

6. Johal JS, Thorp JW, Oyer CE 1998 Neonatal hemochromatosis, renal tubular dysgenesis, and hypocalvaria in a neonate. Pediatr Dev Pathol 1:433-437

7. Morris S, Akima S, Dahlstrom JE, Ellwood D, Kent A, Falk MC 2004 Renal tubular dysgenesis and neonatal hemochromatosis without pulmonary hypoplasia. Pediatr Nephrol 19:341-344

8. Allanson JE, Hunter AG, Mettler GS, Jimenez C 1992 Renal tubular dysgenesis: a not uncommon autosomal recessive syndrome: a review. Am J Med Genet 43:811-814

9. Whitington PF 2006 Fetal and infantile hemochromatosis. Hepatology 43:654-660

10. Gomez RA, Norwood VF 1995 Developmental consequences of the reninangiotensin system. Am J Kidney Dis 26:409-431

11. Guron G, Friberg P 2000 An intact renin-angiotensin system is a prerequisite for normal renal development. J Hypertens 18:123-137

12. Pryde PG, Sedman AB, Nugent CE, Barr M Jr 1993 Angiotensin-converting enzyme inhibitor fetopathy. J Am Soc Nephrol 3:1575-1582

13. Stec DE, Davisson RL, Haskell RE, Davidson BL, Sigmund CD 1999 Efficient liver-specific deletion of a floxed human angiotensinogen transgene by adenoviral delivery of Cre recombinase in vivo. J Biol Chem 274:21285-21290

14. Whitington PF, Malladi P, Melin-Aldana H, Azzam R, Mack CL, Sahai A 2005 Expression of osteopontin correlates with portal biliary proliferation and fibrosis in biliary atresia. Pediatr Res 57:837-844

15. Fleming S, Lindop GB, Gibson AA 1985 The distribution of epithelial membrane antigen in the kidney and its tumours. Histopathology 9:729-739

16. Oberg KC, Pestaner JP, Bielamowicz L, Hawkins EP 1999 Renal tubular dysgenesis in twin-twin transfusion syndrome. Pediatr Dev Pathol 2:25-32

17. Tanguay RM, Valet JP, Lescault A, Duband JL, Laberge C, Lettre F, Plante M 1990 Different molecular basis for fumarylacetoacetate hydrolase deficiency in the two clinical forms of hereditary tyrosinemia (type I). Am J Hum Genet 47:308-316

18. Morris BJ, Iwamoto HS, Reid IA 1979 Localization of angiotensinogen in rat liver by immunocytochemistry. Endocrinology 105:796-800

19. Sahai A, Malladi P, Pan X, Paul R, Melin-Aldana H, Green RM, Whitington PF 2004 Obese and diabetic $\mathrm{db} / \mathrm{db}$ mice develop marked liver fibrosis in a model of nonalcoholic steatohepatitis: role of short-form leptin receptors and osteopontin. Am J Physiol Gastrointest Liver Physiol 287:G1035-G1043

20. Hyde DM, Tyler NK, Plopper CG 2007 Morphometry of the respiratory tract: avoiding the sampling, size, orientation, and reference traps. Toxicol Pathol $35: 41-48$

21. Weibel ER, Hsia CC, Ochs M 2007 How much is there really? Why stereology is essential in lung morphometry. J Appl Physiol 102:459-467 
22. Genest DR, Lage JM 1991 Absence of normal-appearing proximal tubules in the fetal and neonatal kidney: prevalence and significance. Hum Pathol 22:147-153

23. Gribouval O, Gonzales M, Neuhaus T, Aziza J, Bieth E, Laurent N, Bouton JM, Feuillet F, Makni S, Ben Amar H, Laube G, Delezoide AL, Bouvier R, Dijoud F, Ollagnon-Roman E, Roume J, Joubert M, Antignac C, Gubler MC 2005 Mutations in genes in the renin-angiotensin system are associated with autosomal recessive renal tubular dysgenesis. Nat Genet 37:964-968

24. Piper JM, Ray WA, Rosa FW 1992 Pregnancy outcome following exposure to angiotensin-converting enzyme inhibitors. Obstet Gynecol 80:429-432

25. Friberg P, Sundelin B, Bohman SO, Bobik A, Nilsson H, Wickman A, Gustafsson H, Petersen J, Adams MA 1994 Renin-angiotensin system in neonatal rats: induction of a renal abnormality in response to ACE inhibition or angiotensin II antagonism. Kidney Int 45:485-492

26. Olson AL, Perlman S, Robillard JE 1990 Developmental regulation of angiotensinogen gene expression in sheep. Pediatr Res 28:183-185
27. Tufro-McReddie A, Romano LM, Harris JM, Ferder L, Gomez RA 1995 Angiotensin II regulates nephrogenesis and renal vascular development. Am J Physiol 269:F110-F115

28. Bernstein J, Barajas L 1994 Renal tubular dysgenesis: evidence of abnormality in the renin-angiotensin system. J Am Soc Nephrol 5:224-227

29. Avner ED, Sweeney WE Jr, Piesco NP, Ellis D 1985 Growth factor requirements of organogenesis in serum-free metanephric organ culture. In Vitro Cell Dev Bio 21:297-304

30. Ghevaert C, Wilcox DA, Fang J, Armour KL, Clark MR, Ouwehand WH, Williamson LM 2008 Developing recombinant HPA-1a-specific antibodies with abrogated Fcgamma receptor binding for the treatment of fetomaternal alloimmune thrombocytopenia. J Clin Invest 118:2929-2938

31. Rand EB, Karpen SJ, Kelly S, Mack CL, Malatack JJ, Sokol RJ, Whitington PF 2009 Treatment of neonatal hemochromatosis with exchange transfusion and intravenous immunoglobulin. J Pediatr 155:566-571 